\title{
Bilateral Inverted Papilloma: A Rare Rhinological Pathology
}

\author{
${ }^{1}$ Thavakumar Subramaniam, ${ }^{2}$ Ayham Shamis, ${ }^{3}$ Taleb Barghouthi, ${ }^{4}$ Naishadh Patil
}

\section{ABSTRACT}

Inverted papilloma is a benign tumor occurring in the head and neck. A large proportion of reported cases are unilateral, however, in rare instances bilateral disease is seen. This may lead to misdiagnosis.

Keywords: Benign tumor, Bilateral, Inverted papilloma, Rhinology.

How to cite this article: Subramaniam T, Shamis A, Barghouthi T, Patil N. Bilateral Inverted Papilloma: A Rare Rhinological Pathology. Clin Rhinol An Int J 2015;8(2):58-59.

\section{Source of support: Nil \\ Conflict of interest: None}

\section{INTRODUCTION}

Papilloma is a benign epithelial neoplasia that accounts for about $4 \%$ of nasal tumors with an incidence of $1.5 / 100,000$. It is estimated that $99 \%$ of cases are unilateral. ${ }^{1}$ Inverted papilloma (IP) originates from the lateral part of the nasal cavity, and will have a secondary effect on the osteomeatal complex (OMC) near the sinuses. In fact, it is uncommon to find it in the nasal sinuses and its occurrence in the sinuses is found in about $5 \%$ of cases. ${ }^{1-4}$ Epidemiologically, inverted papilloma has a male to female predominance of five to one and is more prevalent in white people, toward their 5 th decade of life. ${ }^{4}$ Despite being benign, IP has the ability of local invasion, high growth potential and high recurrence. A total of $10 \%$ of patients who have an IP with atypia are associated with squamous cell carcinoma. Clinical features are usually a vague nasal obstruction, olfactory problems, recurrent sinusitis and epistaxis. Reference diagnosis is usually by computed tomography (CT) scan, magnetic resonance imaging (MRI) and histopathology and the mainstay of treatment is surgical resection.

\footnotetext{
${ }^{1}$ Trainee Registrar, ${ }^{2,3}$ Registrar, ${ }^{4}$ Consultant

${ }^{1}$ Department of Otolaryngology, St James Hospital, Dublin Ireland

${ }^{2-4}$ Department of ENT, Sligo Regional Hospital, Sligo, Ireland
}

Corresponding Author: Thavakumar Subramaniam, Trainee Registrar, Department of Otolaryngology, St James Hospital Dublin, Ireland, Phone: 00353851586454, e-mail: tksorl@ outlook.com

\section{CASE REPORT}

A 74 years old male was seen in the otolaryngology outpatient department (OPD) complaining of a year long history of nasal blockage, rhinorrhea and frontal headaches. His symptoms are constant with no seasonal variation and denied any significant epistaxis or symptoms of olfactory dysfunction. He denied any significant sinonasal symptoms or therapy prior to this and was otherwise medically fit. The patient's primary care physician referred him to our department after having failed a compliant trial of conservative medical therapy in the community and the persistent nature of his symptoms. Initial outpatient assessment that included a flexible nasoendoscopic examination revealed bilateral grade three nasal polyposis.

A CT scan of the paranasal sinuses reported bilateral nasal polyposis with bilateral maxillary sinus opacification, mucosal thickening and bilateral OMC obstruction. There was also mucosal thickening of the ethmoidal, frontal and sphenoidal sinuses (Figs 1 and 2).

We listed the patient for an examination under anesthesia (EUA) of the bilateral nasal cavity and biopsy. We elected to obtain tissue histology prior listing the patient for a formal functional endoscopic sinus surgery (FESS) as we were suspicious that the nature of onset of patient's symptoms and CT sinus findings were indicative of a more sinister pathology. Magnetic resonance imaging done confirmed soft-tissue enhancement within nasal cavity but bilateral maxillary sinus opacification to be secondary to mucosal thickening and fluid level.

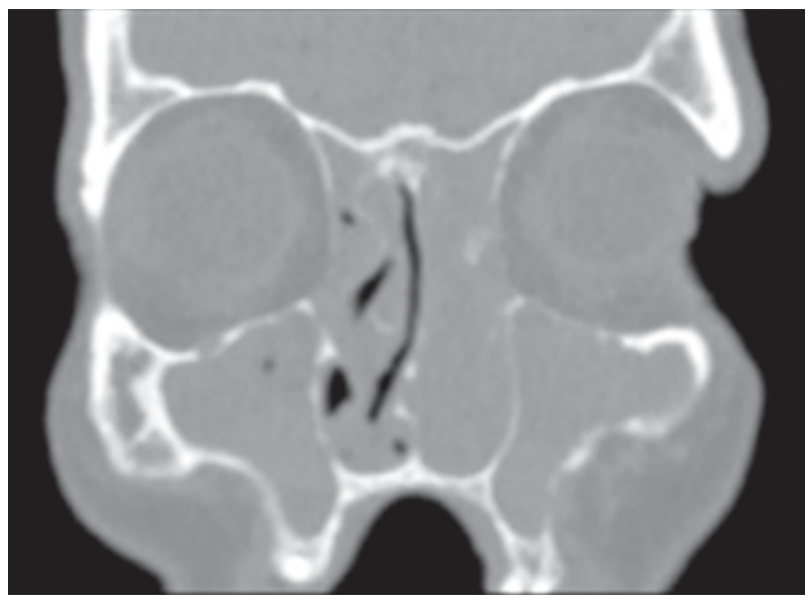

Fig. 1: Coronal paranasal sinus CT showing bilateral opacification of the maxillary sinus 


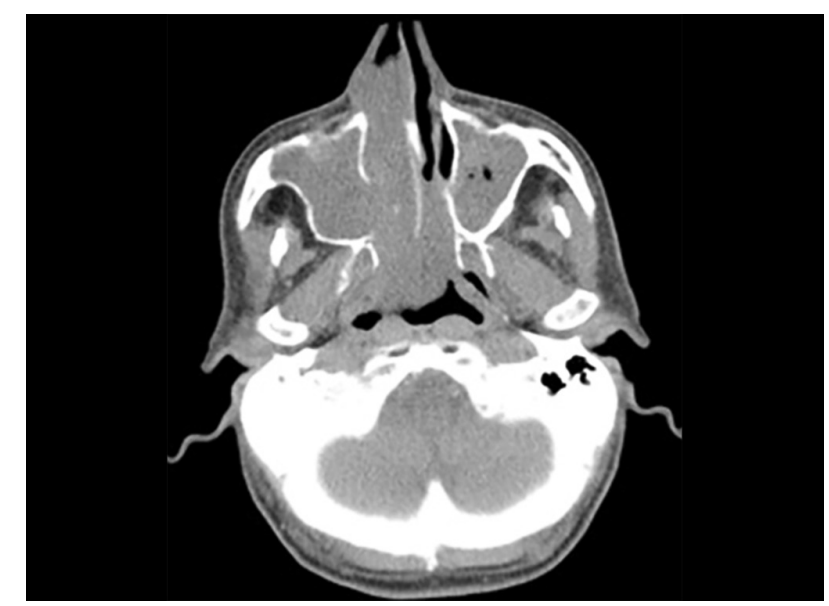

Fig. 2: Axial cuts of the paranasal sinus CT showing a softtissue mass within bilateral nasal cavities

Intraoperative endoscopic evaluation was keeping with the initial outpatient endoscopic findings of grade three bilateral nasal polyposis and biopsies were taken. The procedure was uneventful and histology revealed bilateral nasal inverted papilloma without cell atypia or malignancy. The case was referred on to a tertiary head and neck oncology unit and evaluation. Multidisciplinary team (MDT) decision was for FESS at our institute.

Patient underwent the second definitive procedure for excision of bilateral nasal inverted papilloma via endoscopic approach. Rediscussion at the MDT following surgery showed all surgical margins to be clear and radiotherapy was not advocated as there was no malignant cell detected. The patient was discharged to the OPD for long-term follow-up. Patient currently attends for biannual evaluation and remains disease and symptom free.

\section{DISCUSSION}

Inverted papilloma is a benign tumor of the nasal and paranasal sinuses and etiology of this rare pathology remains unknown. ${ }^{1,2,4}$ Inverted papilloma is also known as Ewing papilloma, transitional cells papilloma, Schneider papilloma and also, transitional cells benign tumor. ${ }^{1,2,4}$

From our literature review, we find a widely accepted theory of etiology of IP is Schneider membrane that forms the nasal cavity and paranasal sinus mucosa undergo cellular changes that predisposes the cells to neoplastic alteration. Other proposed etiologies are inflammatory, infectious, chemical and toxin exposure. ${ }^{3,4}$ Epstein barr virus (EBV) and human papilloma virus (HPV) type 6 and 11 have been implicated in $14 \%$ of cases of IP. ${ }^{4}$

The clinical presentation of IP is usually unilateral nasal obstruction and rarely rhinorrhea, epistaxis, olfactory dysfunction and frontal headaches. ${ }^{1,2,4}$ Delayed presentation may result in symptoms relating to local extension and destruction of tumor. Orbital extension is often like to result in proptosis and orbital vascular or neuromuscular dysfunction leading to diplopia or vision loss. Nasopharyngeal, pterygopalantine and intracranial extension have all been reported. ${ }^{1,2,4}$

Clinical evaluation includes standard head and neck examination with flexible nasoendoscopic evaluation. ${ }^{4}$ Computed tomography scan of the nasal and paranasal sinuses areessential ininvestigating all sinonasal pathology currently, however, histology is often required to confirm the diagnosis. ${ }^{4}$ Magnetic resonance imaging gives better delineation of soft-tissue anatomy and tumor extension. ${ }^{4}$

Surgical resection remains the mainstay of treatment. Prior to FESS, operative management often involved lateral rhinotomy, middle facial open procedures or medial maxillectomy., ${ }^{5,6}$ With current advancements on FESS and microdebridement surgery, minimally invasive surgery is an option. However, minimally invasive surgery should be considered in cases where tumor burden is small, unilateral disease and nonmalignant disease. ${ }^{7}$ The role of radiotherapy as adjuvant therapy remains a controversial. It is indicated in the case of an inoperable IP tumor, malignant transformation or for extensive recurrence of disease. ${ }^{2,8,9}$

Inverted papilloma is a rare rhinology oncological disease with bilateral disease certainly rarer in occurrence. Clinical evaluation, radiological studies and definitive histology remain the main tools for diagnosis. Surgical resection remains the treatment of choice and we certainly advocate MDT approach and tertiary referral center opinion if available.

\section{REFERENCES}

1. Vrabec DP. The inverted Schneiderian papilloma: 25-year study. Laryngoscope 1994;104:582-608.

2. Tsue TT, Bailet JW, Barlow DW, Makielski KH. Bilateral sinusal papilloma in aplasic maxilar sinuses. Am J Otolaryngol 1997;18(4):263-268.

3. Phillips PP, Gustafson RO, Facer GW. The clinical behavior of inverting papilloma of the nose and paranasal sinuses: report of 112 cases and review of the literature. Laryngoscope 1990;100:463-469.

4. Dolgin S, Zaveri V, Casiano R, Maniglia AJ. Different options of treatment of inverting papilloma of the nose and the paranasal sinus: a report of 41 cases. Laryngoscope 1992 Mar;102(3): 231-236.

5. Ringertz N. Pathology of malignant tumors arising in the nasal and paranasal cavities and maxilla. Acta Otolaryngol 1938;27:31-42.

6. Myers EN, Fernau JL, Johnson JT. Management of inverted papilloma. Laryngoscope 1990;100:481-490.

7. Stankiewicz JA, Girs SJ. Endoscopic surgical treatment of nasal and paranasal sinus inverted papilloma. Otolaryngol Head Neck Surg 1993;109:988-995.

8. Weissler MC, Montgomery WW, Montgomery SK. Inverted papiloma. Ann Otol Rhinol Laryngol 1986;95:215-221.

9. Krouse JH. Development of a staging system for inverted papilloma. Laryngoscope 2000;110:965-968. 